\title{
Higgs production in two-photon process and transition form factor
}

\section{Norihisa Watanabe and Yoshimasa Kurihara}

High Energy Accelerator Research Organization (KEK)

Tsukuba, Ibaragi 305-0801, Japan

E-mail: norihisaepost.kek.ip yoshimasa.kuriharaekek.ip

\section{Tsuneo Uematsu}

Institute for Liberal Arts and Sciences, Kyoto University, Kyoto 606-8501, Japan

and Maskwa Institute, Kyoto Sangyo University, Kyoto 603-8555, Japan

E-mail: uematsuescphys.kyoto-u.ac.ip

\section{Ken Sasaki*}

Dept. of Physics, Faculty of Engineering, Yokohama National University

Yokohama 240-8501, Japan

E-mail: sasakidynu.ac.ip

\begin{abstract}
We investigate the SM Higgs boson production in $e \gamma$ collision. The electroweak one-loop contributions to the scattering amplitude for $e \gamma \rightarrow e H$ are calculated. Particularly, we are interested in the contribution from the two-photon fusion process which is described by the transition form factor. We analyze the differential cross section for $e \gamma \rightarrow e H$ when both the initial electron and photon beams are fully polarized and examine the feasibility to observe the transition form factor of the Higgs boson.
\end{abstract}

The XXI International Workshop High Energy Physics and Quantum Field Theory,

June 23-June 30, 2013

Saint Petersburg Area, Russia

\footnotetext{
* Speaker.
} 


\section{Introduction}

After a Higgs boson with mass about $125 \mathrm{GeV}$ was discovered by ATLAS and CMS at LHC [U], a plan for building a new accelerator facility, a linear $e^{+} e^{-}$collider, which offers much cleaner experimental collisions, is attracting growing attention [■]. Along with $e^{+} e^{-}$collider, other options such as $e^{-} e^{-}, e^{-} \gamma$ and $\gamma \gamma$ colliders have been also discussed [B, 团, []]. In this talk, I report our recent study of the Standard Model (SM) Higgs boson $(H)$ production in $e^{-} \gamma$ collider. We examine the reaction $e^{-} \gamma \rightarrow e^{-} H$ at one-loop level. Particularly, we are interested in the study of the two-photon fusion process which is described by the transition form factor. The differential cross section for $e^{-} \gamma \rightarrow e^{-} H$ is analyzed for each case of polarizations of the initial electron and photon beams.

\section{Higgs production in two-photon process and transition form factor}

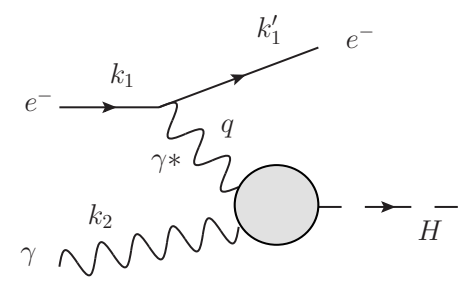

Figure 1: Higgs production in two-photon process

We examine the reaction,

$$
e^{-}\left(k_{1}\right)+\gamma\left(k_{2}\right) \rightarrow e^{-}\left(k_{1}^{\prime}\right)+H\left(p_{h}\right) .
$$

Since $k_{2}$ is the momentum of a real photon, we have $k_{2}^{2}=0$ and $k_{2}^{\beta} \varepsilon_{\beta}\left(k_{2}\right)=0$, where $\varepsilon_{\beta}\left(k_{2}\right)$ is the photon polarization vector. We set $q=k_{1}-k_{1}^{\prime}$. Assuming that the electrons are massless so that $k_{1}^{2}=k_{1}^{\prime 2}=0$, we introduce the following Mandelstam variables:

$$
s=\left(k_{1}+k_{2}\right)^{2}, \quad t=\left(k_{1}-k_{1}^{\prime}\right)^{2}, \quad u=\left(k_{1}-p_{h}\right)^{2}=m_{h}^{2}-s-t,
$$

where $m_{h}$ is the Higgs boson mass. The relevant Feynman diagrams for this reaction start at the one-loop level. Particularly we are interested in the two-photon $\left(\gamma^{*} \gamma\right.$ fusion) process shown in Fig.W. The Higgs production through fusion process $\gamma^{*} \gamma \rightarrow H$ is just opposite to the decay process $H \rightarrow \gamma^{*} \gamma$, which was discussed in Ref. [目] (see, for example, Ref.[四] for the on-shell decay $H \rightarrow$ $\gamma \gamma)$. Since the couplings of Higgs to fermions are proportional to the fermion mass, we only consider the top-quark for the charged fermion loop diagrams. The calculation of the one-loop $\gamma^{*} \gamma$ fusion diagrams is similar to the one for $H \rightarrow \gamma \gamma$. We work in unitary gauge using dimensional regularization which respects electromagnetic gauge invariance.

We obtain the contribution from the one-loop $\gamma^{*} \gamma$ fusion diagrams to the gauge invariant scattering amplitude for $e \gamma \rightarrow e H$ as follows:

$$
A_{\gamma \gamma}=\left(\frac{e^{3} g}{16 \pi^{2}}\right)\left[\bar{u}\left(k_{1}^{\prime}\right) \gamma_{\mu} u\left(k_{1}\right)\right] \frac{1}{t}\left(g^{\mu \beta}-\frac{2 k_{2}^{\mu} q^{\beta}}{m_{h}^{2}-t}\right) \varepsilon_{\beta}\left(k_{2}\right) F_{\gamma \gamma}(t)
$$


with

$$
F_{\gamma \gamma}(t)=\frac{2 m_{t}^{2}}{m_{W}} N_{c} Q_{t}^{2} S_{(T)}^{\gamma \gamma}\left(t, m_{t}^{2}, m_{h}^{2}\right)-m_{W} S_{(W)}^{\gamma \gamma}\left(t, m_{W}^{2}, m_{h}^{2}\right)
$$

where $e$ and $g$ are the electromagnetic coupling and the weak gauge coupling, respectively, and $N_{c}=3$ and $Q_{t}=\frac{2}{3} \cdot S_{(T)}^{\gamma \gamma}$ and $S_{(W)}^{\gamma \gamma}$ are contributions from loops of top quark (with mass $m_{t}$ ) and $W$ boson (with mass $m_{W}$ ), respectively, and expressed in terms of the Passarino-Veltman two- and three-point scalar integrals $B_{0}$ 's and $C_{0}[[]]$ as,

$$
\begin{aligned}
S_{(T)}^{\gamma \gamma}\left(t, m_{t}^{2}, m_{h}^{2}\right)= & 2+\frac{2 t}{m_{h}^{2}-t}\left[B_{0}\left(m_{h}^{2} ; m_{t}^{2}, m_{t}^{2}\right)-B_{0}\left(t ; m_{t}^{2}, m_{t}^{2}\right)\right] \\
S_{(W)}^{\gamma \gamma}\left(t, m_{W}^{2}, m_{h}^{2}\right)= & 6+\frac{m_{h}^{2}-t}{m_{W}^{2}}-\frac{m_{h}^{2} t}{2 m_{W}^{4}}+\left\{4 m_{t}^{2}-m_{h}^{2}+t\right\} C_{0}\left(m_{h}^{2}, 0, t ; m_{t}^{2}, m_{t}^{2}, m_{t}^{2}\right), \\
& +\frac{t\left(12 m_{W}^{4}+2 m_{W}^{2}\left(m_{h}^{2}-t\right)-m_{h}^{2} t\right)}{2 m_{W}^{4}\left(m_{h}^{2}-t\right)}\left[B_{0}\left(m_{h}^{2} ; m_{W}^{2}, m_{W}^{2}\right)-B_{0}\left(t ; m_{W}^{2}, m_{W}^{2}\right)\right] \\
& +\left\{\frac{t\left(m_{h}^{2}-2 t\right)}{m_{W}^{2}}+12 m_{W}^{2}-6 m_{h}^{2}+6 t\right\} C_{0}\left(m_{h}^{2}, 0, t ; m_{W}^{2}, m_{W}^{2}, m_{W}^{2}\right) .
\end{aligned}
$$

A dimensionless quantity $G_{\gamma \gamma}(t) \equiv F_{\gamma \gamma}(t) /\left(\frac{t-m_{h}^{2}}{2 m_{W}}\right)$ may be considered as a transition form factor of the Higgs boson. In the limit $t \rightarrow 0, G_{\gamma \gamma}(t)$ reduces to

$$
G_{\gamma \gamma}(0)=N_{c} Q_{t}^{2} F_{1 / 2}+F_{1},
$$

where $F_{1 / 2}$ and $F_{1}$ are the top-quark and W-boson loop contributions to $H \rightarrow \gamma \gamma$ decay amplitude given in Eq.(2.17) of Ref.[四]. The W-loop contribution $\left|m_{W} S_{(W)}^{\gamma \gamma}\right|$ is much larger in magnitude than the top-quark contribution $\left|\frac{2 m_{t}^{2}}{m_{W}} N_{c} Q_{t}^{2} S_{(T)}^{\gamma \gamma}\right|$ and grows with $-t$. Thus, $G_{\gamma \gamma}(t)$, the sum of top-quark and W-boson contributions, grows with $-t$. Actually, it grows as $\log ^{2} \frac{-t}{m_{W}^{2}}$ for large $-t$.

\section{Contributions from other diagrams}

There are other one-loop diagrams which contribute to the reaction (2. (1). They are $Z^{*} \gamma$ fusion diagrams, " $W-v_{e}$ " diagrams (Fig.[Z) and " $Z-e$ " diagrams (Fig.[1). The one-loop $Z^{*} \gamma$ fusion diagrams are obtained from $\gamma^{*} \gamma$ fusion diagrams by replacement of the photon propagator with that of $Z$ boson (with mass $m_{Z}$ ). Their contribution is expressed as

$$
A_{Z \gamma}=\left(\frac{e g^{3}}{16 \pi^{2}}\right)\left[\bar{u}\left(k_{1}^{\prime}\right) \gamma_{\mu}\left(f_{Z e}+\gamma_{5}\right) u\left(k_{1}\right)\right] \frac{1}{t-m_{Z}^{2}}\left(g^{\mu \beta}-\frac{2 k_{2}^{\mu} q^{\beta}}{m_{h}^{2}-t}\right) \varepsilon_{\beta}\left(k_{2}\right) F_{Z \gamma}(t)
$$

with

$$
F_{Z \gamma}(t)=-\frac{m_{t}^{2}}{8 m_{W} \cos ^{2} \theta_{W}} N_{c} Q_{t} f_{Z t} S_{(T)}^{Z \gamma}\left(t, m_{t}^{2}, m_{h}^{2}\right)+\frac{m_{W}}{4} S_{(W)}^{Z \gamma}\left(t, m_{W}^{2}, m_{h}^{2}\right)
$$

where $f_{Z e}$ and $f_{Z t}$ are the strength of vector part of the $Z$ coupling to electron and top quark, respectively, and are given by $f_{Z e}=-1+4 \sin ^{2} \theta_{W}$ and $f_{Z t}=1-\frac{8}{3} \sin ^{2} \theta_{W}$. We find $S_{(T)}^{Z \gamma}=S_{(T)}^{\gamma \gamma}$ and $S_{(W)}^{Z \gamma}=S_{(W)}^{\gamma \gamma}$. 

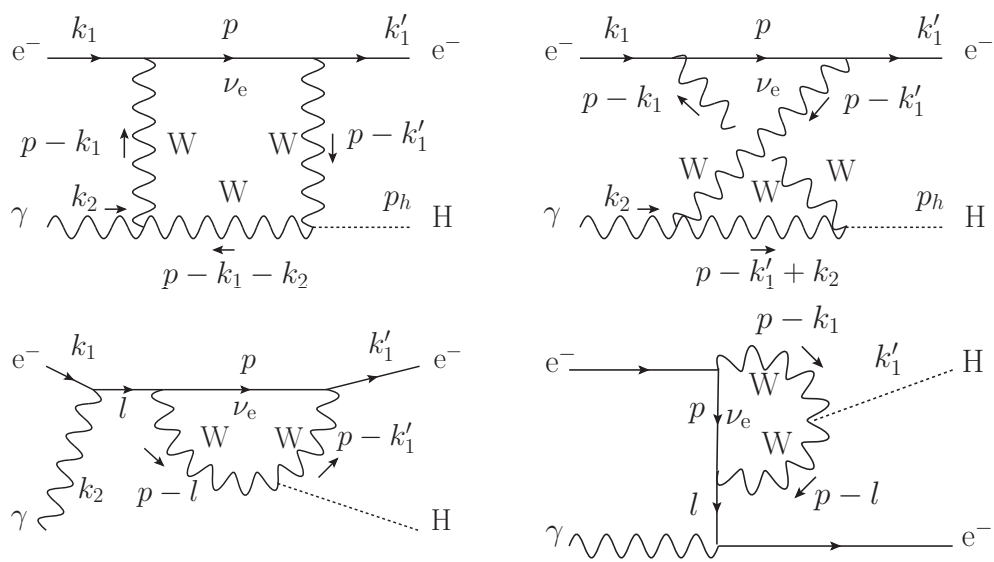

Figure 2: " $W-v_{e}$ " diagrams

The Feynman diagrams involving $W$ boson and electron neutrino, which are shown in Fig.】, yield the " $W v_{e}$ " amplitude which is written as,

$$
A_{W v_{e}}=\left(\frac{e g^{3}}{16 \pi^{2}}\right) \frac{m_{W}}{4}\left[\bar{u}\left(k_{1}^{\prime}\right) F_{\left(W v_{e}\right) \beta}\left(1-\gamma_{5}\right) u\left(k_{1}\right)\right] \varepsilon\left(k_{2}\right)^{\beta}
$$

where the factor $\left(1-\gamma_{5}\right)$ is due to the $e-v-W$ vertex. The factor $F_{\left(W v_{e}\right) \beta}$ is written in a gauge invariant form as

$$
F_{\left(W v_{e}\right) \beta}=\left(\frac{2 k_{1_{\beta}} k_{2}}{s}-\gamma_{\beta}\right) S_{\left(k_{1}\right)}^{W v_{e}}\left(s, t, m_{h}^{2}, m_{W}^{2}\right)+\left(\frac{2 k_{1_{\beta}}^{\prime} k_{2}}{u}+\gamma_{\beta}\right) S_{\left(k_{1}^{\prime}\right)}^{W v_{e}}\left(s, t, m_{h}^{2}, m_{W}^{2}\right),
$$

where $S_{\left(k_{1}\right)}^{W v_{e}}\left(s, t, m_{h}^{2}, m_{W}^{2}\right)$ and $S_{\left(k_{1}^{\prime}\right)}^{W v_{e}}\left(s, t, m_{h}^{2}, m_{W}^{2}\right)$ are expressed in terms of the two-, three- and fourpoint scalar integrals $B_{0}$ 's, $C_{0}$ 's and $D_{0}$ 's, which will be reported elsewhere [Q].
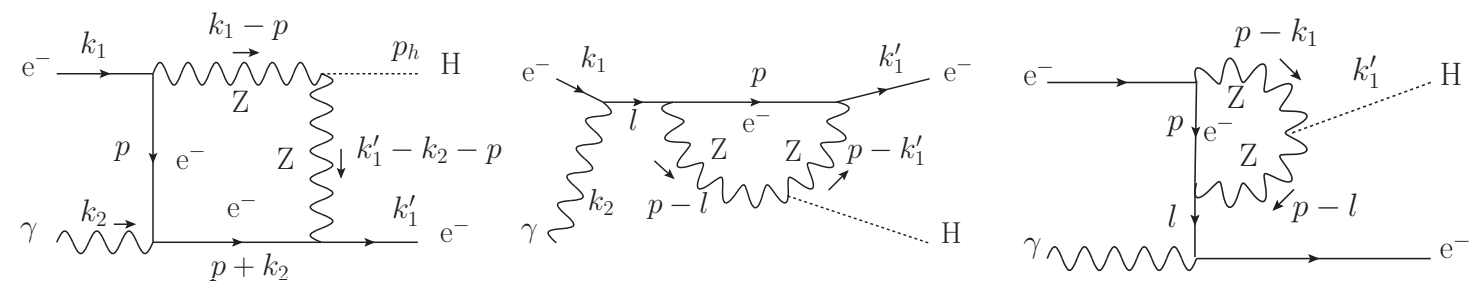

Figure 3: "Z- $e$ " diagrams

Finally, the " $Z-e$ " diagrams shown in Fig.[1 give the following amplitude,

$$
A_{Z e}=\left(\frac{e g^{3}}{16 \pi^{2}}\right)\left(-\frac{m_{Z}}{16 \cos ^{3} \theta_{W}}\right) \times\left[\bar{u}\left(k_{1}^{\prime}\right) F_{(Z e) \beta}\left(f_{Z e}+\gamma_{5}\right)^{2} u\left(k_{1}\right)\right] \varepsilon\left(k_{2}\right)^{\beta},
$$

where the factor $\left(f_{Z e}+\gamma_{5}\right)^{2}$ arises from the $Z$ boson coupling to electrons. The factor $F_{(Z e) \beta}$ is written in a gauge invariant form as

$$
F_{(Z e) \beta}=\left(\frac{2 k_{1_{\beta}} k_{2}}{s}-\gamma_{\beta}\right) S_{\left(k_{1}\right)}^{Z e}\left(s, t, m_{h}^{2}, m_{Z}^{2}\right)+\left(\frac{2 k_{1_{\beta}}^{\prime} k_{2}}{u}+\gamma_{\beta}\right) S_{\left(k_{1}^{\prime}\right)}^{Z e}\left(s, t, m_{h}^{2}, m_{Z}^{2}\right)
$$

where $S_{\left(k_{1}\right)}^{Z e}\left(s, t, m_{h}^{2}, m_{Z}^{2}\right)$ and $S_{\left(k_{1}^{\prime}\right)}^{Z e}\left(s, t, m_{h}^{2}, m_{Z}^{2}\right)$ are expressed in terms of the two-, three- and fourpoint scalar integrals $B_{0}$ 's, $C_{0}$ 's and $D_{0}$ 's, which will be reported elsewhere [Q]. 


\section{Higgs production cross section}

One of the advantages of the linear collider machines is that we can acquire good polarization for the initial colliding beams. Let us consider the Higgs production process (2ل when both the initial electron and photon beams are fully polarized. We denote the initial electron helicity as $P_{e}= \pm 1$ and photon helicity as $P_{\gamma}= \pm 1$. The differential cross section for $e \gamma \rightarrow e H$ with the initial electron and photon helicity $P_{e}$ and $P_{\gamma}$ is expressed by,

$$
\frac{d \sigma_{(e \gamma \rightarrow e H)}\left(s, P_{e}, P_{\gamma}\right)}{d t}=\frac{1}{16 \pi s^{2}} \times\left\{\sum_{\text {final electron spin }}\left|A\left(P_{e}, P_{\gamma}\right)\right|^{2}\right\}
$$

where $A\left(P_{e}, P_{\gamma}\right)=A_{\gamma \gamma}\left(P_{e}, P_{\gamma}\right)+A_{Z \gamma}\left(P_{e}, P_{\gamma}\right)+A_{W v_{e}}\left(P_{e}, P_{\gamma}\right)+A_{Z e}\left(P_{e}, P_{\gamma}\right)$.

The angular momentum conservation requires that, apart from the photon propagator which appears as $\frac{1}{t}$, an overall factor $t$ arises in the differential cross section. Also the differential cross section for the initial beams with $P_{e} P_{\gamma}=-1$ should vanish as $u \rightarrow 0$ (or $t \rightarrow t_{\max }=m_{h}^{2}-s$ ).

When an initial electron is polarized with polarization $P_{e}$, we modify $u\left(k_{1}\right)$ as

$$
u\left(k_{1}\right) \rightarrow \frac{1+P_{e} \gamma_{5}}{2} u\left(k_{1}\right) .
$$

The polarization tensor of a circularly polarized photon moving in the $+z$-direction is given by

$$
\varepsilon\left(k_{2}, \pm 1\right)_{\alpha}^{*} \varepsilon\left(k_{2}, \pm 1\right)_{\beta}=-\frac{1}{2} g_{\alpha \beta} \pm \frac{i}{2}\left(g_{\alpha 1} g_{\beta 2}-g_{\alpha 2} g_{\beta 1}\right) \text {. }
$$

Using Eqs.(4.2) - (4.3), we evaluate $\sum_{\text {final electron spin }}\left|A\left(P_{e}, P_{\gamma}\right)\right|^{2}$ and obtain the differential cross section for $e \gamma \rightarrow e H$ for each case of polarizations of the initial electron and photon beams. In order to see the relative contributions from $\gamma^{*} \gamma$ fusion, $Z^{*} \gamma$ fusion, " $W-v_{e}$ " and " $Z-e$ " diagrams, we evaluate the differential cross section given in Eq.(A.]) replacing $A\left(P_{e}, P_{\gamma}\right)$ with $A_{\gamma \gamma}\left(P_{e}, P_{\gamma}\right)$, $A_{Z \gamma}\left(P_{e}, P_{\gamma}\right), A_{W v_{e}}\left(P_{e}, P_{\gamma}\right)$ and $A_{Z e}\left(P_{e}, P_{\gamma}\right)$, respectively. We obtain

$$
\begin{aligned}
& \frac{d \sigma_{(\gamma \gamma)}\left(P_{e}, P_{\gamma}\right)}{d t}= \frac{1}{16 \pi s^{2}}\left(\frac{e^{3} g}{16 \pi^{2}}\right)^{2}\left(-\frac{1}{t}\right) F_{\gamma \gamma}(t)^{2}\left\{\frac{s^{2}+u^{2}}{(s+u)^{2}}+P_{\gamma} P_{e}\left(1-\frac{2 u}{s+u}\right)\right\}, \\
& \frac{d \sigma_{(Z \gamma)}\left(P_{e}, P_{\gamma}\right)}{d t}= \frac{1}{16 \pi s^{2}}\left(\frac{e g^{3}}{16 \pi^{2}}\right)^{2} \frac{-t}{\left(t-m_{Z}^{2}\right)^{2}} F_{Z \gamma}(t)^{2} \\
& \times\left\{\left(f_{Z e}^{2}+2 P_{e} f_{Z e}+1\right) \frac{s^{2}+u^{2}}{(s+u)^{2}}+P_{\gamma}\left(P_{e} f_{Z e}^{2}+2 f_{Z e}+P_{e}\right)\left(1-\frac{2 u}{s+u}\right)\right\}, \\
& \frac{d \sigma_{\left(W v_{e}\right)}\left(P_{e}, P_{\gamma}\right)}{d t}= \frac{1}{16 \pi s^{2}}\left(\frac{e g^{3}}{16 \pi^{2}}\right)^{2} \frac{m_{W}^{2}}{8}(-t)\left(1-P_{e}\right)\left\{\left[\left|S_{\left(k_{1}\right)}^{W v_{e}}\left(s, t, m_{h}^{2}, m_{W}^{2}\right)\right|^{2}+\left|S_{\left(k_{1}^{\prime}\right)}^{W v_{e}}\left(s, t, m_{h}^{2}, m_{W}^{2}\right)\right|^{2}\right]\right. \\
&\left.+P_{\gamma}\left[-\left|S_{\left(k_{1}\right)}^{W v_{e}}\left(s, t, m_{h}^{2}, m_{W}^{2}\right)\right|^{2}+\left|S_{\left(k_{1}^{\prime}\right)}^{W v_{e}}\left(s, t, m_{h}^{2}, m_{W}^{2}\right)\right|^{2}\right]\right\}, \\
& \frac{d \sigma_{(Z e)}\left(P_{e}, P_{\gamma}\right)}{d t}= \frac{1}{16 \pi s^{2}}\left(\frac{e g^{3}}{16 \pi^{2}}\right)^{2}\left(\frac{m_{Z}}{16 \cos ^{3} \theta_{W}}\right)^{2}(-t) \\
& \quad \times\left\{\left(f_{Z e}^{4}+4 P_{e} f_{Z e}^{3}+6 f_{Z e}^{2}+4 P_{e} f_{Z e}+1\right)\left[\left|S_{\left(k_{1}\right)}^{Z e}\left(s, t, m_{h}^{2}, m_{Z}^{2}\right)\right|^{2}+\left|S_{\left(k_{1}^{\prime}\right)}^{Z e}\left(s, t, m_{h}^{2}, m_{Z}^{2}\right)\right|^{2}\right]\right. \\
&\left.\quad+P_{\gamma}\left(P_{e} f_{Z e}^{4}+4 f_{Z e}^{3}+6 P_{e} f_{Z e}^{2}+4 f_{Z e}+P_{e}\right)\left[\left|S_{\left(k_{1}\right)}^{Z e}\left(s, t, m_{h}^{2}, m_{Z}^{2}\right)\right|^{2}-\left|S_{\left(k_{1}^{\prime}\right)}^{Z e}\left(s, t, m_{h}^{2}, m_{Z}^{2}\right)\right|^{2}\right]\right\} .(4.7)
\end{aligned}
$$


In order to calculate the differential cross section for $e \gamma \rightarrow e H$ given in Eq.(4.]), we also need to evaluate the interference terms among the four amplitudes $A_{\gamma \gamma}\left(P_{e}, P_{\gamma}\right), A_{Z \gamma}\left(P_{e}, P_{\gamma}\right), A_{W v_{e}}\left(P_{e}, P_{\gamma}\right)$ and $A_{Z e}\left(P_{e}, P_{\gamma}\right)$.
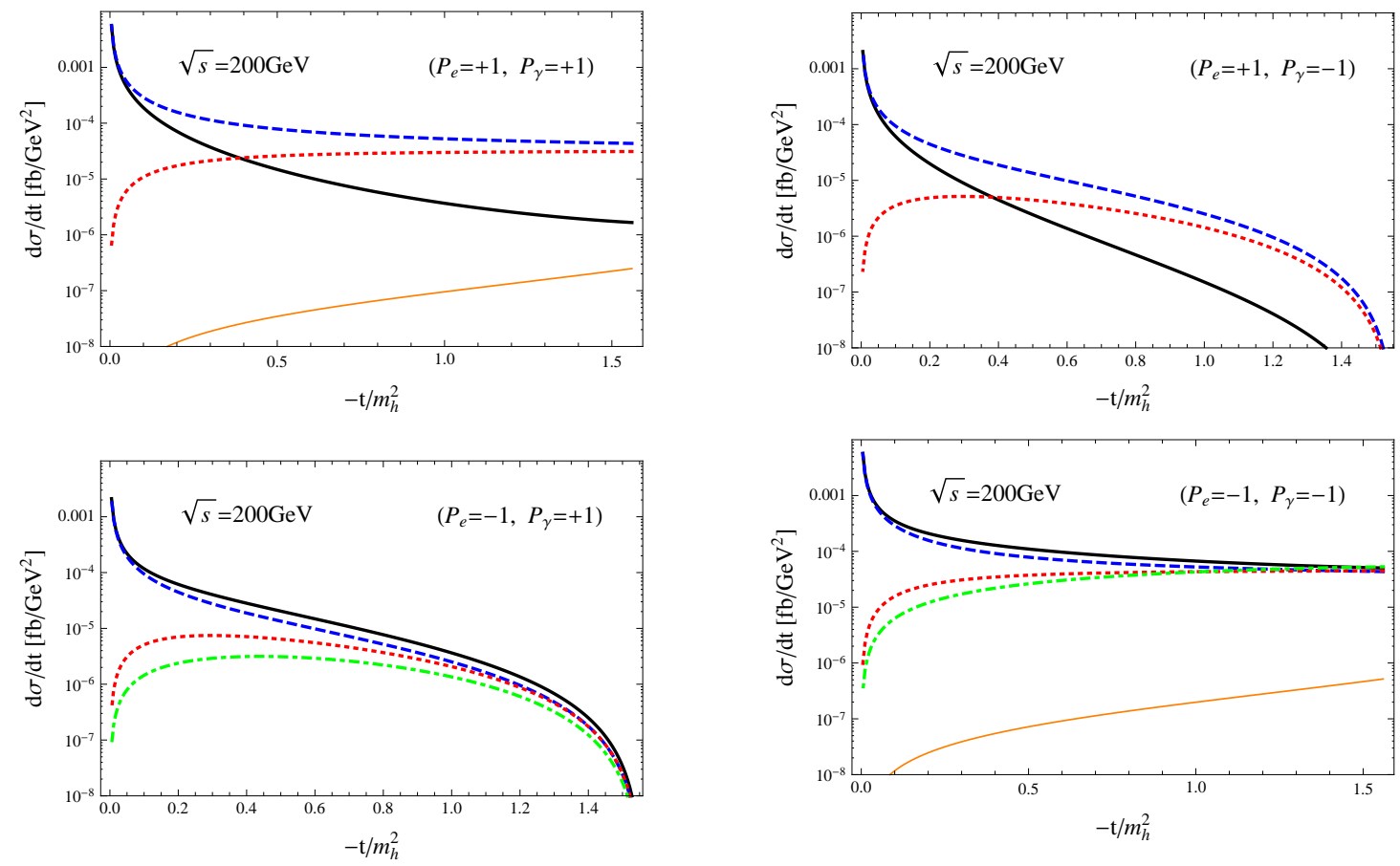

Figure 4: The differential cross section for Higgs production $d \sigma_{(e \gamma \rightarrow e H)} / d t$ (black solid line) together with $d \sigma_{(\gamma \gamma)} / d t$ (blue dashed line), $d \sigma_{(Z \gamma)} / d t$ (red dotted line), $d \sigma_{\left(W v_{e}\right)} / d t$ (green dotdashed line) and $d \sigma_{(Z e)} / d t$ (orange thin solid line) as a function of $-t / m_{H}^{2}$ with $\sqrt{s}=200 \mathrm{GeV}$ for four cases of polarizations of the initial electron and photon beams, $\left(P_{e}=+1, P_{p}=+1\right),\left(P_{e}=+1, P_{p}=-1\right),\left(P_{e}=-1, P_{p}=+1\right)$ and $\left(P_{e}=-1, P_{p}=-1\right)$. In the plots of $\left(P_{e}=+1, P_{p}=-1\right)$ and $\left(P_{e}=-1, P_{p}=+1\right), d \sigma_{(Z e)} / d t$ is too small and out of the plot range.

For numerical analysis we choose the mass parameters and the coupling constants as follows:

$$
\begin{aligned}
& m_{h}=125 \mathrm{GeV}, \quad m_{t}=173 \mathrm{GeV}, \quad m_{Z}=91 \mathrm{GeV}, \quad m_{W}=80 \mathrm{GeV} \\
& \cos \theta_{W}=\frac{m_{W}}{m_{Z}}, \quad e^{2}=4 \pi \alpha_{e m}=\frac{4 \pi}{128}, \quad g=\frac{e}{\sin \theta_{W}} .
\end{aligned}
$$

The electromagnetic constant $e^{2}$ is chosen to be the value at the scale of $m_{Z}$. We plot these differ-

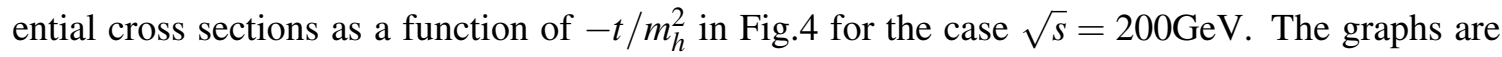
shown for each case of polarizations of the initial electron and photon beams. First we find the contribution from the "Z-e" diagrams is negligibly small for all cases compared with those from the other three. Also we see that when $P_{e} P_{\gamma}=-1$ all the graphs diminish as $t \rightarrow t_{\max }=m_{h}^{2}-s$.

For the case of polarizations $P_{e}=-1$ and $P_{\gamma}= \pm 1$, a dominant contribution comes from the $\gamma^{*} \gamma$ fusion diagrams for smaller $|t|$, more specifically, up to $-t / m_{h}^{2} \leq 1$. This is due to the factor $(-1 / t)$ in the expression (4.4) for $d \sigma_{(\gamma \gamma)} / d t$, which arises as $(-t) \times\left(1 / t^{2}\right)$ with $1 / t$ coming from the photon propagator. For $1<-t / m_{h}^{2}<1.5$, the contributions to the differential cross section from $\gamma^{*} \gamma$ fusion, $Z^{*} \gamma$ fusion and " $W-v_{e}$ " diagrams become the same order, and at $-t / m_{h}^{2}>1.5$, 
the contribution of " $W-v_{e}$ " diagrams prevails over the other two. For $P_{e}=-1$ and $P_{\gamma}= \pm 1$, the interference between $A_{\gamma \gamma}$ and $A_{Z \gamma}$ works constructively, while the one between $A_{\gamma \gamma}$ and $A_{W v_{e}}$ works destructively and its effect becomes large at $-t / m_{h}^{2}>1.5$. Thus the values of $d \sigma_{e \gamma \rightarrow e H} / d t$ become smaller than those of $d \sigma_{\gamma \gamma} / d t, d \sigma_{Z \gamma} / d t$ and $d \sigma_{W v_{e}} / d t$.

For the electron polarization $P_{e}=+1$, no contribution comes from " $W-v_{e}$ " diagrams. The interference between $A_{\gamma \gamma}$ and $A_{Z \gamma}$ works destructively and its effect is large even from small $-t / m_{h}^{2}$ for $P_{e}=+1$ and $P_{\gamma}= \pm 1$. Therefore, $d \sigma_{(e \gamma \rightarrow e H)} / d t$ decreases rather rapidly as $-t / m_{h}^{2}$ increases.

From this analysis, we have seen that, for the case of polarizations $P_{e}=-1$ and $P_{\gamma}= \pm 1$, the contribution of the $\gamma^{*} \gamma$ fusion diagrams to $d \sigma_{(e \gamma \rightarrow e H)} / d t$ is dominant in the region where $-t / m_{h}^{2}$ is smaller than 1 . Therefore, the transition form factor of the Higgs boson via $\gamma^{*} \gamma$ fusion is measurable once an $e \gamma$ colliding machine is constructed.

\section{Summary}

We have investigated the SM Higgs boson production in $e^{-} \gamma$ collision. The electroweak oneloop contributions to the scattering amplitude for $e \gamma \rightarrow e H$ were calculated. We have studied the transition form factor of the Higgs boson which arises from the two-photon fusion process. From the analysis for the differential cross section when both the initial electron and photon beams are polarized, we see that, for $P_{e}=-1$ and $P_{\gamma}= \pm 1$, the contribution of the $\gamma^{*} \gamma$ fusion diagrams dominates in the region for $-t / m_{h}^{2} \leq 1$, and, therefore, we conclude that the transition form factor of the Higgs boson is measurable in an $e \gamma$ collider.

I thank the organizers of QFTHEP'2013 for the pleasant atmosphere during the symposium.

\section{References}

[1] ATLAS Collaboration, Phys. Lett. B716 (2012) 1-29; CMS Collaboration, Phys. Lett. B716 (2012) 30-61.

[2] http://www.linearcollider.org/cms.

[3] A. De Roeck, "Physics at a $\gamma \gamma, e \gamma$ and $e^{-} e^{-}$Option for a Linear Collider", arXiv:hep-ph/0311138 (2003).

[4] S. A. Bogacz et al., "SAPPHiRE: a Small $\gamma \gamma$ Higgs Factory", arXiv:1208.2827 [physics.acc-ph] (2012).

[5] I. F. Ginzburg and M. Krawczyk, "Testing Higgs Physics at the Photon Collider”, arXiv:1310.5881 [hep-ph] (2013).

[6] J. C. Romao and S. Andringa, Eur. Phys. J. C 7 (1999) 631.

[7] J. F. Gunion, H. E. Haber, G. Kane and S. Dawson, “The Higgs Hunter's Guide”(Addison-Wesley, 1990).

[8] G. Passarino and M. Veltman, Nucl. Phys. B160 (1979) 151.

[9] N. Watanabe, Y. Kuriharam, T. Uematsu and K. Sasaki, in preparation. 